\title{
The forward-backward asymmetry for massive bottom quarks at the $Z$ peak at next-to-next-to-leading order QCD
}

\author{
Werner Bernreuther, ${ }^{a, 1}$ Long Chen, ${ }^{a}$ Oliver Dekkers, ${ }^{b}$ Thomas Gehrmann ${ }^{c}$ and \\ Dennis Heisler ${ }^{a}$ \\ ${ }^{a}$ Institut für Theoretische Teilchenphysik und Kosmologie, RWTH Aachen University, \\ 52056 Aachen, Germany \\ ${ }^{b}$ PRISMA Cluster of Excellence and Institut für Physik, Johannes-Gutenberg-Universität Mainz, \\ 55099 Mainz, Germany \\ ${ }^{c}$ Physik-Institut, Universität Zürich, \\ CH-8057 Zürich, Switzerland \\ E-mail: breuther@physik.rwth-aachen.de, \\ algeochen@physik.rwth-aachen.de, dekkers@uni-mainz.de, \\ thomas.gehrmann@uzh.ch, heisler@physik.rwth-aachen.de
}

ABSTRACT: We compute the order $\alpha_{s}^{2}$ QCD corrections to the $b$-quark forward-backward asymmetry in $e^{+} e^{-} \rightarrow b \bar{b}$ collisions at the $Z$ boson resonance, taking the non-zero mass of the $b$ quark into account. We determine these corrections with respect to both the $b$-quark axis and the thrust axis definition of the asymmetry. We compute also the distributions of these axes with respect to the electron beam. If one neglects the flavor singlet contributions to the $b$-quark asymmetry, as was done in previous computations for massless $b$ quarks, then the second-order QCD corrections for $m_{b} \neq 0$ are smaller in magnitude than the corresponding corrections for $m_{b}=0$. Including the singlet contributions slightly increases the magnitude of the corrections. The massive $\alpha_{s}^{2}$ corrections to the $b$-quark forwardbackward asymmetry slightly diminish the well-known tension between the bare $b$-quark asymmetry and the standard model fit from $2.9 \sigma$ to $2.6 \sigma$.

KEYWORDS: NLO Computations

ARXIV EPRINT: 1611.07942

\footnotetext{
${ }^{1}$ Corresponding author.
} 


\section{Contents}

1 Introduction 1

2 The forward-backward asymmetry 2

2.1 Unexpanded and expanded asymmetry to order $\alpha_{s}^{2} \quad 3$

2.2 Quark axis and thrust axis 5

2.3 Set-up of our calculation 5

3 Contributions to order $\alpha_{s}^{2} \quad 6$

3.1 Non-singlet contributions 6

3.2 Triangle contributions $\quad 6$

$\begin{array}{lll}3.3 & \text { Singlet contributions } & 8\end{array}$

3.4 Contributions from the $b \bar{b} b \bar{b}$ final state $\quad 8$

4 Numerical results for the $b$-quark asymmetry at the $Z$ peak $\quad 9$

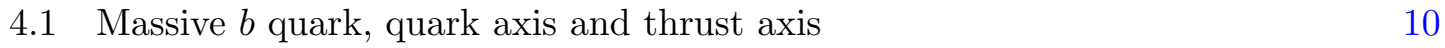

$\begin{array}{lll}4.2 & \text { Approaching the limit of massless } b \text { quarks } & 12\end{array}$

$\begin{array}{lll}4.3 & \text { Discussion } & 14\end{array}$

5 Conclusions $\quad 15$

\section{Introduction}

Forward-backward asymmetries $A_{\mathrm{FB}}^{f}$ are precision observables for the determination of the neutral current couplings of leptons and quarks $f$ in the reactions $e^{+} e^{-} \rightarrow f \bar{f}$. As far as quarks are concerned, the most precisely known asymmetry is that of the $b$ quark at the $Z$ resonance, which was measured with an accuracy of 1.7 percent $[1,2]$. Among the measured set of precision observables at the $Z$ pole, $A_{\mathrm{FB}}^{b}$ shows a relatively large deviation, about 2.9 $\sigma$, from the respective Standard Model (SM) fit. So far, it has not been clarified whether this deviation is due to underestimated experimental and/or theoretical uncertainties or whether it is a hint of new physics.

At a future linear or circular $e^{+} e^{-}$collider [3-5], precision determinations of electroweak parameters will again involve forward-backward asymmetries. If such a collider will be operated at the $Z$ peak, an accuracy of about 0.1 percent may be reached for these observables $[6,7]$.

This has motivated us to compute $A_{\mathrm{FB}}^{b}$ for massive $b$ quarks produced at the $Z$ resonance to second order in the QCD coupling $\alpha_{s}$. The following SM radiative corrections to the lowest-order quark forward-backward asymmetry associated with quark-antiquark production in $e^{+} e^{-}$collisions are known. The fully massive next-to-leading order (NLO) 
electroweak and QCD corrections were determined by [8-10] and by [11-13], respectively. The full next-to-next-to-leading order (NNLO) QCD corrections, i.e., the contributions of $\alpha_{s}^{2}$ to this asymmetry, were recently published for the top quark in $t \bar{t}$ production above the production threshold ${ }^{1}$ in $[14,15]$. For $b$ quarks, the order $\alpha_{s}^{2}$ corrections were calculated so far only in the limit of vanishing $b$-quark mass [17-20]. As pointed out in [19], the forwardbackward asymmetry for a specific massless quark flavor $Q$ is not infrared (IR) safe if the direction that specifies the forward and backward hemisphere is defined by the direction of flight of the quark $Q$ or by the thrust direction. In these cases $A_{\mathrm{FB}}^{Q}$ is affected in the limit $m_{Q} \rightarrow 0$ by logarithmic mass divergences $\sim \ln m_{Q}$. These logarithmically enhanced terms were taken into account in [19] in their computations of $A_{\mathrm{FB}}^{Q}(Q=b, c)$ both with respect to the quark and the thrust axis. In [20] a definition of $A_{\mathrm{FB}}^{Q}$ based on the jet axis was given that is IR finite in the limit $m_{Q} \rightarrow 0$, and $A_{\mathrm{FB}}^{Q}$ was calculated for massless quarks to second order in $\alpha_{s}$. The definition used in [20] is an application of the infrared-safe definition of a flavored quark jet given in [21].

The contributions to $A_{\mathrm{FB}}^{Q}$ at NNLO QCD from the two-parton final state $(Q \bar{Q})$ and from the sum of the three- and four-parton final states $(Q \bar{Q} g$ and $Q \bar{Q} g g, Q \bar{Q} q \bar{q}, Q \bar{Q} Q \bar{Q})$ to this observable are separately IR finite [17]. The order $\alpha_{s}^{2}$ two-parton contributions to $A_{\mathrm{FB}}^{Q}$ were computed in [22]. Here we calculate the full order $\alpha_{s}^{2}$ QCD corrections to the $b$-quark forward-backward asymmetry, both for the quark axis and the thrust axis definition, for massive $b$ quarks to leading order in the electroweak couplings at the $Z$ resonance.

Our paper is organized as follows. In section 2 we define the forward-backward asymmetry $A_{\mathrm{FB}}^{Q}$ to order $\alpha_{s}^{2}$, unexpanded and expanded in the QCD coupling, and we briefly describe our computational approach which is based on [15]. In section 3 we classify the various contributions to the $b$-quark asymmetry into flavor non-singlet, flavor singlet, and triangle terms, with particular attention payed to the contribution of the $b \bar{b} b \bar{b}$ final state, following [19]. Our results for the $b$-quark asymmetry to order $\alpha_{s}^{2}$ and $m_{b} \neq 0$ are presented in section 4. We compare our results with the QCD corrections that were used in previous data analyses $[1,2,23,24]$. Moreover, we consider a subset of order $\alpha_{s}^{2}$ contributions to $A_{\mathrm{FB}}^{b}$ that remain finite in the limit $m_{b} \rightarrow 0$ [19]. We compute the contributions of this subset for a sequence of decreasing $b$-quark masses. Extrapolating to $m_{b}=0$ we find agreement with the massless results of $[18,19]$. We conclude in section 5 .

\section{The forward-backward asymmetry}

Our computational approach applies to the production of any massive quark-antiquark pair in $e^{+} e^{-}$collisions,

$$
e^{+} e^{-} \rightarrow \gamma^{*}, Z^{*} \rightarrow Q \bar{Q}+X
$$

to lowest order in the electroweak couplings and to second order in the QCD coupling $\alpha_{s}$. To this order, the cross section of the reaction (2.1) receives contributions from the two-parton $Q \bar{Q}$ state (at Born level, to order $\alpha_{s}$, and to order $\alpha_{s}^{2}$ ), the three-parton state

\footnotetext{
${ }^{1}$ The forward-backward asymmetry for $t \bar{t}$ production at the Tevatron is also known at NNLO QCD [16].
} 
$Q \bar{Q} g$ (to order $\alpha_{s}$ and to order $\alpha_{s}^{2}$ ), and the four-parton states $Q \bar{Q} g g, Q \bar{Q} q \bar{q}$, and above the $4 Q$ threshold from $Q \bar{Q} Q \bar{Q}$ (to order $\alpha_{s}^{2}$ ). The computation of the differential cross section to order $\alpha_{s}^{2}$ was set up in [15] within the antenna subtraction framework. We use the formulas of [15] and apply it to the production of $b$ quarks at the $Z$ resonance.

The forward-backward asymmetry $A_{\mathrm{FB}}$ for a massive quark $Q$ is defined by ${ }^{2}$

$$
A_{\mathrm{FB}}=\frac{N_{F}-N_{B}}{N_{F}+N_{B}}
$$

where $N_{F}$ and $N_{B}$ are the number of quarks $Q$ observed in the forward and backward hemisphere, respectively. Forward and backward hemispheres are defined with respect to a certain infrared-safe axis. Common choices, which we will use in this paper, are the direction of flight of the heavy quark $Q$ or the direction of the oriented thrust axis. These axes are infrared and collinear safe for massive quarks; thus $A_{\mathrm{FB}}$ is computable in perturbation theory.

The asymmetry (2.2) can be expressed in terms of the cross section for the inclusive production of a massive quark $Q$ [19], i.e. $d \sigma\left(e^{+} e^{-} \rightarrow Q+X\right) / d x_{Q} d \cos \theta$, where $\theta$ is the angle between the the electron three-momentum and the axis defining the forward hemisphere and $x_{Q}=2 E_{Q} / \sqrt{s}$. Here $E_{Q}$ is the energy of $Q$ and $s$ is the squared $e^{+} e^{-}$ center-of-mass (c.m.) energy. Both $\theta$ and $E_{Q}$ are defined in the $e^{+} e^{-}$c.m. frame. With this distribution one can define forward and backward cross sections

$$
\sigma_{F}=\int_{0}^{1} d \cos \theta \int_{x_{0}}^{1} d x_{Q} \frac{d \sigma}{d x_{Q} d \cos \theta}, \quad \sigma_{B}=\int_{-1}^{0} d \cos \theta \int_{x_{0}}^{1} d x_{Q} \frac{d \sigma}{d x_{Q} d \cos \theta},
$$

and symmetric and antisymmetric cross section $\sigma_{S}$ and $\sigma_{A}$,

$$
\sigma_{S}=\sigma_{F}+\sigma_{B}, \quad \sigma_{A}=\sigma_{F}-\sigma_{B} .
$$

Here $x_{0}=2 m_{Q} / \sqrt{s}$ where $m_{Q}$ is the mass of $Q$. With (2.4) the forward-backward asymmetry (2.2) can be expressed as

$$
A_{\mathrm{FB}}=\frac{\sigma_{A}}{\sigma_{S}} .
$$

Notice that above the threshold for $Q \bar{Q} Q \bar{Q}$ production the Feynman diagrams associated with this process contribute with a multiplicity factor two both to $\sigma_{S}$ and $\sigma_{A}$ because this final state contains two quarks $Q$.

\subsection{Unexpanded and expanded asymmetry to order $\alpha_{s}^{2}$}

The forward-backward asymmetry belongs to the class of observables that can be computed at the level of unresolved partons. A number of individual terms in the following perturbative expansions are, however, IR divergent and understood to be regulated with antenna subtraction terms as outlined in [15].

\footnotetext{
${ }^{2}$ For ease of notation we drop here and in the following the superscript $Q$ in $A_{\mathrm{FB}}$ as we will exclusively consider $b$ quarks.
} 
To order $\alpha_{s}^{2}$ the symmetric and antisymmetric cross sections receive the following contributions from unresolved partons:

$$
\sigma_{A, S}=\sigma_{A, S}^{(2,0)}+\sigma_{A, S}^{(2,1)}+\sigma_{A, S}^{(3,1)}+\sigma_{A, S}^{(2,2)}+\sigma_{A, S}^{(3,2)}+\sigma_{A, S}^{(4,2)}+\mathcal{O}\left(\alpha_{s}^{3}\right),
$$

where the first number in the superscripts $(i, j)$ denotes the number of partons in the respective final state and the second one the order of $\alpha_{s}$. Inserting (2.6) into (2.5) we get for $A_{\mathrm{FB}}$ to second order in $\alpha_{s}$ :

$$
A_{\mathrm{FB}}\left(\alpha_{s}^{2}\right)=\frac{\sigma_{A}^{(2,0)}+\sigma_{A}^{(2,1)}+\sigma_{A}^{(3,1)}+\sigma_{A}^{(2,2)}+\sigma_{A}^{(3,2)}+\sigma_{A}^{(4,2)}}{\sigma_{S}^{(2,0)}+\sigma_{S}^{(2,1)}+\sigma_{S}^{(3,1)}+\sigma_{S}^{(2,2)}+\sigma_{S}^{(3,2)}+\sigma_{S}^{(4,2)}}=A_{\mathrm{FB}}^{\mathrm{LO}} C_{2},
$$

where

$$
A_{\mathrm{FB}}^{\mathrm{LO}}=\frac{\sigma_{A}^{(2,0)}}{\sigma_{S}^{(2,0)}},
$$

is the forward-backward asymmetry at Born level and $C_{2}$ is the second-order QCD correction factor defined by the ratio on the left of this equation. The unexpanded forwardbackward asymmetry at order $\alpha_{s}$ is denoted by $A_{\mathrm{FB}}\left(\alpha_{s}\right)=A_{\mathrm{FB}}^{\mathrm{LO}} C_{1}$.

A Taylor expansion of (2.7) to second order in $\alpha_{s}$ gives

$$
A_{\mathrm{FB}}^{\mathrm{NNLO}}=A_{\mathrm{FB}}^{\mathrm{LO}}\left[1+A_{1}+A_{2}\right]+\mathcal{O}\left(\alpha_{s}^{3}\right),
$$

where $A_{1}$ and $A_{2}$ are the QCD corrections of $\mathcal{O}\left(\alpha_{s}\right)$ and $\mathcal{O}\left(\alpha_{s}^{2}\right)$, respectively.

$$
\begin{aligned}
& A_{1}=\sum_{i=2,3}\left[\frac{\sigma_{A}^{(i, 1)}}{\sigma_{A}^{(2,0)}}-\frac{\sigma_{S}^{(i, 1)}}{\sigma_{S}^{(2,0)}}\right], \\
& A_{2}=\sum_{i=2,3,4}\left[\frac{\sigma_{A}^{(i, 2)}}{\sigma_{A}^{(2,0)}}-\frac{\sigma_{S}^{(i, 2)}}{\sigma_{S}^{(2,0)}}\right]-\frac{\sigma_{S}^{(2,1)}+\sigma_{S}^{(3,1)}}{\sigma_{S}^{(2,0)}} A_{1} .
\end{aligned}
$$

The expanded NLO asymmetry will be denoted by $A_{\mathrm{FB}}^{\mathrm{NLO}}=A_{\mathrm{FB}}^{\mathrm{LO}}\left(1+A_{1}\right)$.

The unexpanded and expanded second-order forward-backward asymmetries (2.7) and (2.9) differ by terms of order $\alpha_{s}^{3}$. We will evaluate both expressions and the corresponding expressions at order $\alpha_{s}$ in section 4 .

As to the expanded version (2.9) of the forward-backward asymmetry, we recall that the two-parton and the sum of the three- and four-parton contributions to $A_{2}$ are separately infrared (IR) finite, cf. $[17,19,22]$. The $Q \bar{Q}$ contribution to $A_{2}$ is determined by the oneloop [11] and two-loop [25-27] QCD vertex form factors $\gamma^{*}, Z^{*} \rightarrow Q \bar{Q}$ and it was calculated in [22] for massive $b$ and top quarks. The sum of the three- and four-parton contributions to $A_{2}$ could be computed with any NLO method that can handle the IR divergences in the three- and four-parton matrix elements individually. However, for the calculation of the numerator and denominator of the unexpanded asymmetry (2.7) an NNLO IR method is required. We calculate both versions of the $b$-quark forward-backward asymmetry at NNLO with the set-up of [15]. 


\subsection{Quark axis and thrust axis}

As already mentioned above we will use both the $b$-quark direction of flight and the oriented thrust axis for defining the forward and backward hemispheres. If the $b$-quark direction of flight is chosen then $\theta=\theta_{b}=\angle\left(\vec{k}_{1}, \vec{p}_{1}\right)$ in eq. $(2.3)$, where $\vec{k}_{1}$ and $\vec{p}_{1}$ are the three-momenta of the $b$ quark and of the electron, respectively, in the c.m. frame. Yet, an accurate determination of the $b$-quark momentum is impeded by quark fragmentation and decay. In the past, experimental analyses often used the thrust axis as reference axis. For a given $n$-parton event described by a collection of final-state four-momenta $\left\{k_{i}\right\}_{i=1}^{n}$ (related by momentum conservation), the thrust axis is the direction $\vec{n}_{T}$ that maximizes the thrust $T$ defined by $[28,29]$ :

$$
T=\max _{\vec{n}_{T}} \frac{\sum_{i=1}^{n}\left|\vec{k}_{i} \cdot \vec{n}_{T}\right|}{\sum_{i=1}^{n}\left|\vec{k}_{i}\right|}, \quad\left|\vec{n}_{T}\right|=1 .
$$

It can be shown $[30,31]$ that

$$
\vec{n}_{T} \| \sum_{i} \varepsilon_{i} \vec{k}_{i}, \quad \varepsilon_{i} \in\{0, \pm 1\}
$$

which implies that (2.12) is equivalent to the finite maximization problem:

$$
T=\max _{\varepsilon_{i}} \frac{\left|\sum_{i} \varepsilon_{i} \vec{k}_{i}\right|}{\sum_{i}\left|\vec{k}_{i}\right|} .
$$

This formula determines $\vec{n}_{T}$ up to a sign. Its orientation is fixed by requiring $\vec{n}_{T} \cdot \vec{k}_{1}>0$. Thus, if the thrust axis is chosen as reference axis, the forward hemisphere is defined by $\vec{n}_{T} \cdot \vec{p}_{1}>0$. Therefore, in this case $\theta=\theta_{T}=\angle\left(\vec{n}_{T}, \vec{p}_{1}\right)$ in eq. (2.3).

\subsection{Set-up of our calculation}

Because we work to lowest order in the electroweak couplings, each of the various contributions $d \sigma^{(i, j)}$ to the differential $b \bar{b}$ cross section to order $\alpha_{s}^{2}$ listed at the beginning of this section is given, at arbitrary c.m. energy, by the sum of an s-channel $\gamma$ and $Z$-boson contribution and a $\gamma Z$ interference term. The $d \sigma^{(i, j)}$ have the structure

$$
d \sigma^{(i, j)}=\sum_{a=\gamma, Z, \gamma Z} K_{a}^{(j)} L_{a}^{\mu \nu} H_{a, \mu \nu}^{(i, j)} d \Phi_{i}
$$

Here $d \Phi_{i}$ is the $i$-particle phase-space measure, $L_{a}^{\mu \nu}$ denote the lepton tensors (with the boson propagators included), and $H_{a, \mu \nu}^{(i, j)}$ are the antenna-subtracted, i.e., IR finite parton tensors of order $\alpha_{s}^{j}[15]$. Thus the Lorentz contractions and the phase-space integration in (2.15) can be done in $D=4$ dimensions. The first index $i$ in the superscript $(i, j)$ labels the final state, i.e., $i=b \bar{b}, b \bar{b} g, b \bar{b} g g, b \bar{b} q \bar{q}(q=u, d, s, c, b)$. The factors $K_{a}^{(j)}$ contain the electroweak couplings, the flux factor, and the $e^{+} e^{-}$spin-averaging factor. In this work we consider unpolarized $e^{-} e^{+}$collisions. 
The electroweak neutral current couplings are

$$
v_{f}^{Z}=\frac{e}{2 s_{W} c_{W}}\left(T_{f}^{3}-2 s_{W}^{2} e_{f}\right), \quad a_{f}^{Z}=\frac{e}{2 s_{W} c_{W}}\left(-T_{f}^{3}\right), \quad v_{f}^{\gamma}=e e_{f}, \quad a_{f}^{\gamma}=0 .
$$

Here $f$ denotes a quark or the electron, $e_{f}$ and $T_{f}^{3}$ are the charge of $f$ in units of the positron charge $e$ and its weak isospin, respectively, and $s_{W}\left(c_{W}\right)$ are the sine (cosine) of the weak mixing angle $\vartheta_{W}$.

We separate each contribution $(i, j)$ on the right-hand side of (2.15) into a parityeven and -odd term. As we work to lowest order in the electroweak couplings, these terms determine the cross sections (2.4) that are symmetric and antisymmetric under the exchange of $b$ and $\bar{b}$, respectively. For the numerical evaluation of the $d \sigma^{(i, j)}$ we use the approach described in detail in [15]. In section 4 we consider $b \bar{b}$ production exactly at the $Z$ resonance. At this c.m. energy the s-channel $\gamma$ and $\gamma Z$ interference contributions to the $d \sigma^{(i, j)}$ are neglected.

\section{Contributions to order $\alpha_{s}^{2}$}

In this section we briefly discuss the various terms that contribute to the $b \bar{b}$ cross section and, in particular, to the $b$-quark forward-backward asymmetry to order $\alpha_{s}^{2}$. Below we shall compute the $b$-quark asymmetry also for a sequence of decreasing $b$-quark masses in order to compare with the massless results of $[18,19]$. For this comparison it is useful to classify the contributions into flavor non-singlet (NS), flavor singlet (S), and interference or triangle (Tr) terms. We follow here the notation and discussion of [19]. Schematically the differential cross section may be written as

$$
d \sigma=d \sigma_{\mathrm{NS}}+d \sigma_{\mathrm{S}}+d \sigma_{\mathrm{Tr}} .
$$

Flavor singlet and triangle contributions are present only at order $\alpha_{s}^{2}$. The contribution of the $b \bar{b} b \bar{b}$ final state to $A_{\mathrm{FB}}$ deserves special attention and will be discussed in section 3.4 below.

\subsection{Non-singlet contributions}

This class denotes contributions to (2.15) and (3.1) where the electroweak current couples to the $b \bar{b}$ pair. As to the two-parton, i.e., $b \bar{b}$ final state: apart from the LO and NLO diagrams (cf. figure $1 \mathrm{a}$ and $1 \mathrm{~b}$ ), $d \sigma_{\mathrm{NS}}$ receives contributions of the type shown in figure $1 \mathrm{c}$. Non-singlet contributions from the three-parton final state are shown in figure $2 \mathrm{a}$ and $2 \mathrm{~b}$. All the diagrams that correspond to the $b \bar{b} g g$ final state (cf. figure $3 \mathrm{a}$ ) and the square of $b \bar{b} q \bar{q}(q \neq b)$ diagram figure $3 \mathrm{~b}$ belong to this class, too. There are also contributions from the $b \bar{b} b \bar{b}$ final state, see section 3.4.

\subsection{Triangle contributions}

This class involves Feyman diagrams with quark triangles, namely the interference between the diagrams in figure $1 \mathrm{a}$ and $1 \mathrm{~d}$, between the diagrams in figure $2 \mathrm{a}$ and $2 \mathrm{c}$, and between the diagrams in figure $3 \mathrm{~b}$ and $3 \mathrm{c}$. The triangles in figures $1 \mathrm{~d}$ and $2 \mathrm{c}$ represent a sum 


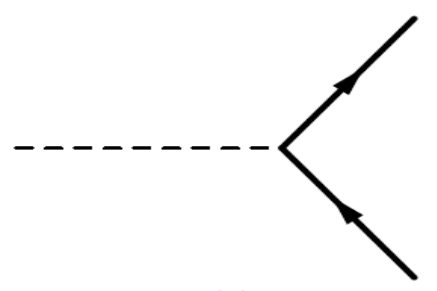

(a)

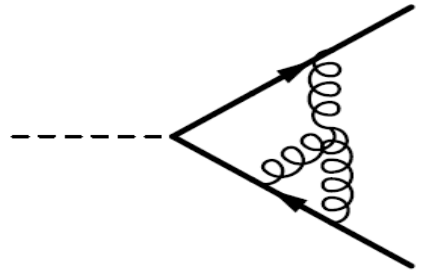

(c)

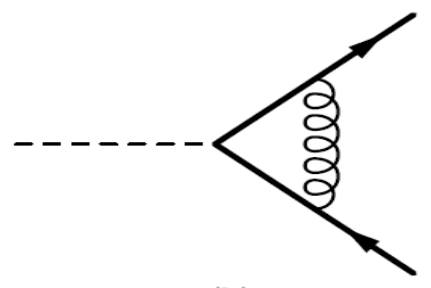

(b)

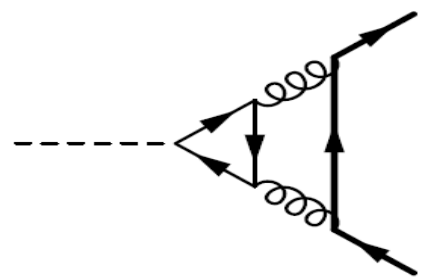

(d)

Figure 1. Examples of diagrams that contribute to the $b \bar{b}$ final state to order $\alpha_{s}^{2}$. The dashed line represents the electroweak neutral current, the thick line the $b$ quark, and the thin line any of the six quarks. The triangle diagrams (d) are summed over the six quark flavors.

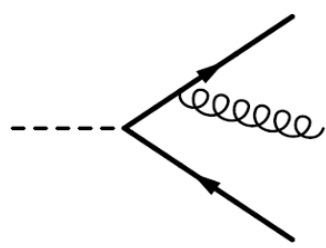

(a)

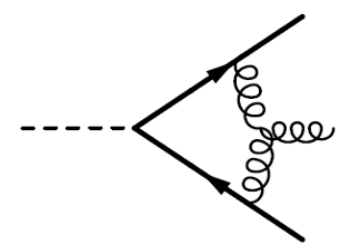

(b)

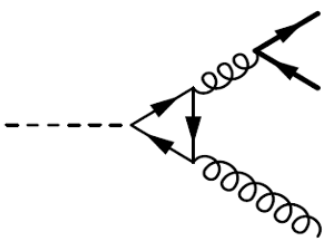

(c)

Figure 2. Examples of diagrams that contribute to the $b \bar{b} g$ final state to order $\alpha_{s}^{2}$. The assignment of the lines is as in figure 1 .

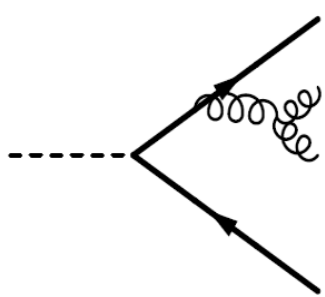

(a)

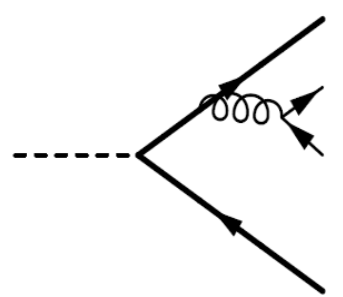

(b)

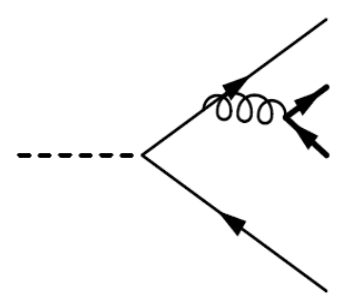

(c)

Figure 3. (a): Examples of diagrams that contribute to the $b \bar{b} g g$ final state at order $\alpha_{s}^{2}$. (b,c): Diagrams that contribute to the $b \bar{b} q \bar{q}(q \neq b)$ final state at order $\alpha_{s}^{2}$.

over all six quark flavors which couple to the respective axial current. We use massless $u, d, c, s$ quarks. Their contributions cancel pairwise because up- and down-type quarks have weak isospin quantum numbers $T_{q}^{3}$ of opposite sign. The non-vanishing contributions to the triangles in figures $1 \mathrm{~d}$ and $2 \mathrm{c}$, that is, the differences between the $b$ - and $t$-quark 
triangles, are ultraviolet and infrared finite. The triangle contributions are non-universal corrections to the leading order $b$-quark forward-backward asymmetry, because they involve electroweak couplings of quarks $q \neq b$.

\subsection{Singlet contributions}

The square of the diagrams figure $3 \mathrm{c}$ belongs to this class. Here the $b \bar{b}$ pair is produced by the splitting of a gluon radiated off a light quark. Only $\sigma_{S}$ receives a contribution from this class, but not $\sigma_{A}$. There is an additional singlet contribution to $\sigma_{S}$ from the $b \bar{b} b \bar{b}$ final state as will be discussed in the next subsection.

\subsection{Contributions from the $b \bar{b} b \bar{b}$ final state}

Four amplitudes $\mathcal{D}_{i}$, where each denotes the sum of the two diagrams shown in figure 4 , are associated with this final state because it contains two $b$ and two $\bar{b}$ quarks. Therefore, as already emphasized above, these diagrams must be counted twice in the calculation of $\sigma_{S}$ and $\sigma_{A}$. In our calculation of the $b$-quark forward-backward asymmetry in section 4.1 we sum these diagrams and take the square, taking into account the multiplicity and statistics factor 2 and $1 / 4$, respectively.

Yet, for our calculation of $A_{\mathrm{FB}}$ for a sequence of decreasing $b$-quark masses, which is done for the purpose of comparing with the massless result of [19], it is necessary to make a subdivision of the $b \bar{b} b \bar{b}$ term as was done in this reference. Ref. [19] distinguishes between (i) contributions that are identical to those of $b \bar{b} q \bar{q}$, figures $3 \mathrm{~b}$ and $3 \mathrm{c}$, but with $q$ being replaced by that $b$ quark that is not triggered on, and (ii) genuine interference terms due to the fact that there are two indistinguishable (anti)quarks in the final state. Group (ii), which is called the E-term in [32], is the color subleading part of the squared $b \bar{b} b \bar{b}$ matrix element. In the following $D_{i j}=\operatorname{Re}\left(\mathcal{D}_{i}^{*} \mathcal{D}_{j}\right)$ where, as already emphasized, $\mathcal{D}_{i}$ is the sum of the diagrams shown in figure 4 . The E-term is given by the sum of the following interferences:

$$
D_{12}, D_{13}, D_{24}, D_{34}
$$

Ref. [19] considers the E-term to be part of the non-singlet contributions. Group (i) can be partitioned into non-singlet, singlet, and triangle contributions. The singlet terms are those where the $b$ quark that is triggered on is produced by a gluon. By convention we assign the momentum $k_{1}$ to this quark. Then the singlet contribution is given by the sum of the terms

$$
D_{33} \text { and } D_{44} \text {. }
$$

The triangle contribution is given by the sum of the terms

$$
D_{14} \text { and } D_{23} \text {. }
$$

These are interferences between diagrams where the $b$ quark with momentum $k_{1}$ couples to the weak current and to the gluon, respectively. The remaining contributions to group (i) are non-singlet contributions.

We come back to this classification in section 4.2 when comparing with [19]. 

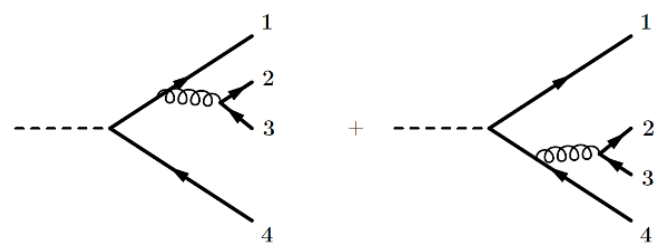

(a) Amplitudes $\mathcal{D}_{i}, i=1, \ldots, 4$.

\begin{tabular}{|c|c|c|c|c|}
\hline Label & $\mathcal{D}_{1}$ & $\mathcal{D}_{2}$ & $\mathcal{D}_{3}$ & $\mathcal{D}_{4}$ \\
\hline 1 & $k_{1}$ & $k_{1}$ & $k_{3}$ & $k_{3}$ \\
2 & $k_{3}$ & $k_{3}$ & $k_{1}$ & $k_{1}$ \\
3 & $k_{4}$ & $k_{2}$ & $k_{4}$ & $k_{2}$ \\
4 & $k_{2}$ & $k_{4}$ & $k_{2}$ & $k_{4}$ \\
\hline
\end{tabular}

(b) Labeling of the quark momenta

Figure 4. Contributions to $e^{+} e^{-} \rightarrow b \bar{b} b \bar{b}$.

\section{Numerical results for the $b$-quark asymmetry at the $Z$ peak}

In this section we present our results for the $b$-quark asymmetry at NNLO QCD and lowest order in the electroweak couplings at the $Z$ resonance, with respect to both the $b$-quark and the thrust direction. As mentioned before, we put the couplings of the virtual photon to the fermions to zero, as we work to lowest order in the electroweak couplings. We emphasize that our set-up allows to compute the $b$-quark asymmetry at arbitrary energies $\sqrt{s} \neq m_{Z}$; i.e., to take into account also the photon exchange and $\gamma-Z$ interference terms.

We use the computational framework of [15], that is, antenna-subtracted renormalized matrix elements with the $b$-quark mass defined in the on-shell scheme and the QCD coupling $\alpha_{s}$ defined in the $\overline{\mathrm{MS}}$ scheme. For the $\overline{\mathrm{MS}}$ mass of the $b$ quark we take the average determined in [33]:

$$
\bar{m}_{b}\left(\bar{m}_{b}\right)=4.18 \pm 0.03 \mathrm{GeV},
$$

which yields the on-shell mass:

$$
m_{b}=4.89 \pm 0.04 \mathrm{GeV} .
$$

For the mass of the top quark that appears in the triangle diagrams figure $1 \mathrm{~d}$ and $2 \mathrm{c}$ we use $m_{t}=173.34 \mathrm{GeV}$. The $u, d, c, s$ quarks are taken to be massless. For the $\overline{\mathrm{MS}}$ coupling of five-flavor QCD we take $\alpha_{s}\left(\mu=m_{Z}\right)=0.118$ where $\mu$ is the renormalization scale. The scale variation, i.e., the renormalization group running of $\alpha_{s}$ is determined with the first three coefficients of the beta function. The sine of the weak mixing angle, $s_{W}$, is fixed by $s_{W}^{2}=1-m_{W}^{2} / m_{Z}^{2}$. With $m_{W}=80.385 \mathrm{GeV}$ and $m_{Z}=91.1876 \mathrm{GeV}$ one gets $s_{W}^{2}=0.2229$. For computing the electroweak couplings of the quarks and the electron we use the $G_{\mu}$ scheme where the electromagnetic coupling is given by $\alpha=\sqrt{2} G_{\mu} m_{W}^{2} s_{W}^{2} / \pi=7.5624 \times 10^{-3}$ with $G_{\mu}=1.166379 \times 10^{-5} \mathrm{GeV}^{-2}$.

As mentioned above the $b \bar{b}$ contribution to the second-order correction $A_{2}$, eq. (2.11), is IR finite. It was computed in [22] with unsubtracted $b \bar{b}$ matrix elements. Here we compute the $b \bar{b}$ contribution to $A_{2}$ with antenna-subtracted matrix elements. As a check of our set-up we calculated this contribution with the input parameters of [22] and found agreement with the numbers given in table 1 of this reference. We checked also that the sum of the three- and four-parton contributions to $A_{2}$ is IR finite. 


\begin{tabular}{|c|c|c|c|c|}
\hline & $1+A_{1}$ & $1+A_{1}+A_{2}$ & $A_{1}$ & $A_{2}$ \\
\hline quark axis: & $0.9710_{-0.0034}^{+0.0028}$ & $0.9587_{-0.0028}^{+0.0026}$ & -0.0290 & -0.0123 \\
\hline thrust axis: & $0.9713_{-0.0026}^{+0.0027}$ & $0.9608_{-0.0025}^{+0.0022}$ & -0.0287 & -0.0105 \\
\hline
\end{tabular}

Table 1. The first- and second-order QCD correction factors defined in (2.9)-(2.11) to the LO $b$-quark forward-backward asymmetry at the $Z$ peak for the input values given in the text and for $\mu=m_{Z}$. The numbers in superscript (subscript) refer to the changes if $\mu=2 m_{Z}\left(\mu=m_{Z} / 2\right)$ is chosen.

\subsection{Massive $b$ quark, quark axis and thrust axis}

With the values of $s_{W}^{2}$ and the bottom mass given above, the tree-level value of the $b$ quark forward-backward asymmetry at $\sqrt{s}=m_{Z}$ is $A_{\mathrm{FB}}^{\mathrm{LO}}=0.1512$. The value of $A_{\mathrm{FB}}^{\mathrm{LO}}$ is very sensitive to the input value of $s_{W}^{2}$ but insensitive to the uncertainty on $m_{b}$ given in (4.2). We are concerned here with the first- and second-order QCD corrections to the LO asymmetry. They are given in table 1 for the expanded version of $A_{\mathrm{FB}}$, both for the quark axis and the thrust axis definition, for the three renormalization scales $\mu=m_{Z} / 2, m_{Z}, 2 m_{Z}$. In this subsection we take into account all contributions discussed in section 3 .

Table 1 shows that the order $\alpha_{s}^{2}$ corrections are significant. For $\mu=m_{Z}$ the ratio $A_{2} / A_{1}$ is $43 \%$ and $37 \%$ for the quark and thrust axis definition, respectively. Variation of the scale as in table 1 changes both the first- and second-order QCD correction factors by about \pm 0.003 with respect to their values at $\mu=m_{Z}$. The fact that inclusion of the second-order correction term $A_{2}$ does not (significantly) reduce the scale uncertainty is not unusual for an observable that is defined as a ratio.

The first- and second-order corrections $A_{1}$ and $A_{2}$ are dominated by the contributions from the three-parton and three- and four-parton final states, respectively. In the limit $m_{b} \rightarrow 0$ the $b \bar{b}$ contribution to $A_{1}$ and the non-singlet $b \bar{b}$ contribution to $A_{2}$ vanish because the chiral non-singlet currents become conserved. Because $m_{b} / m_{Z} \ll 1$ these contributions to $A_{1}$ and $A_{2}$ turn out to be about two orders of magnitude smaller than the three-parton, respectively three- and four-parton contributions. The $b \bar{b}$ triangle contribution to $A_{2}$ (cf. figure 1d) is about one order of magnitude larger than the non-singlet $b \bar{b}$ contribution, but an order of magnitude smaller than those from the three- and four-parton final states.

We have included in the computation of $A_{2}$ given in table 1 also the non-universal corrections $A_{2}^{\text {non-u. }}$ of order $\alpha_{s}^{2}$ that contain the vector and axial vector couplings of quarks $q \neq b$. They are significant. For instance, for the quark axis definition and $\mu=m_{Z}$ we get $A_{2}^{\text {non }-\mathrm{u} .}=-0.00310$ which is $25 \%$ of the total correction $A_{2}$. This number comes about as follows. The two- and the three-parton contribution to $A_{2}^{\text {non-u. }}$, that is, the interference of figure $1 \mathrm{a}$ and $1 \mathrm{~d}$ and of figure $2 \mathrm{a}$ and $2 \mathrm{c}$, is small; it is +0.00085 and +0.00028 , respectively. The dominant part is due to the non-universal contributions from the $b \bar{b} q \bar{q}(q \neq b)$ final state. While the term $\sigma_{A}^{(4,2)} / \sigma_{A}^{(2,0)}$ (cf. eq. (2.11)) of this correction is negligibly small, the term $\sigma_{S}^{(4,2)} / \sigma_{S}^{(2,0)}$ is significant. As a result the contribution of this term to $A_{2}^{\text {non-u. is }}$ -0.00423 . Notice that the value of $A_{2}^{\text {non-u. }}$ depends on the value of $s_{W}^{2}$. 


\begin{tabular}{|cc|c|c|}
\hline & & $a_{1}$ & $a_{2}$ \\
\hline quark axis & $\mu=m_{Z} / 2:$ & 1.544 & 26.67 \\
& $\mu=m_{Z}:$ & 1.544 & 34.84 \\
& $\mu=2 m_{Z}:$ & 1.544 & 43.06 \\
\hline thrust axis & $\mu=m_{Z} / 2:$ & 1.528 & 21.75 \\
& $\mu=m_{Z}:$ & 1.528 & 29.81 \\
& $\mu=2 m_{Z}:$ & 1.528 & 37.98 \\
\hline
\end{tabular}

Table 2. The values of the first- and second-order coefficients $a_{1}$ and $a_{2}$ defined in (4.3) for $\mu=m_{Z} / 2, m_{Z}$, and $2 m_{Z}$.

\begin{tabular}{|c|c|c|}
\hline & $C_{1}$ & $C_{2}$ \\
\hline quark axis: & $0.9722_{-0.0031}^{+0.0025}$ & $0.9594_{-0.0030}^{+0.0026}$ \\
\hline thrust axis: & $0.9725_{-0.0031}^{+0.0025}$ & $0.9614_{-0.0026}^{+0.0023}$ \\
\hline
\end{tabular}

Table 3. The first- and second-order QCD correction factors $C_{1}$ and $C_{2}$ defined in eq. (2.7) and below eq. (2.8).

Next we represent the expanded version of the b-quark asymmetry, both for the quark and the thrust axis, in the form:

$$
A_{\mathrm{FB}}^{\mathrm{NNLO}}=A_{\mathrm{FB}}^{\mathrm{LO}}\left[1-a_{1} \frac{\alpha_{s}}{2 \pi}-a_{2}\left(\frac{\alpha_{s}}{2 \pi}\right)^{2}\right]
$$

Table 2 contains the values of the coefficients $a_{1}$ and $a_{2}$ extracted from the values of $A_{1}$ and $A_{2}$ of table 1.

Monte-Carlo simulations or measurements of the $b$-quark forward-backward asymmetry at the $Z$ peak can also be compared with perturbative computations where the ratio $\sigma_{A} / \sigma_{S}$ is not expanded. In this case the order $\alpha_{s}$ and order $\alpha_{s}^{2}$ correction factors $C_{1}$ and $C_{2}$ apply that are defined in (2.7) and below (2.8). Their values are given in table 3.

The spread between the second-order expanded and unexpanded correction factors may be viewed, in addition or alternatively to scale variations, as an indication of the order of magnitude of the uncalculated higher-order corrections. The comparison of the values of $1+A_{1}+A_{2}$ and $C_{2}$ for fixed $\mu$ given in table 1 and 3 shows that both for the quark and the thrust axis definition the spread between these correction factors is significantly smaller than the change of these terms due to scale variations. This indicates that the perturbative calculation of the $b$-quark forward-backward asymmetry is reliable.

Finally we display in figure 5 the distributions of $\cos \theta_{b}$ and $\cos \theta_{T}$ to order $\alpha_{s}^{2}$, where $\theta_{b}$ $\left(\theta_{T}\right)$ is the angle between the $b$-quark direction (oriented thrust direction) and the electron beam. Here we use the schematic notation $d \sigma_{\mathrm{NLO}}=d \sigma_{\mathrm{LO}}+d \sigma_{1}$ and $d \sigma_{\mathrm{NNLO}}=d \sigma_{\mathrm{NLO}}+d \sigma_{2}$. The plots show that the order $\alpha_{s}^{2}$ corrections to these un-normalized distributions are small and these corrections reduce the scale uncertainties. 

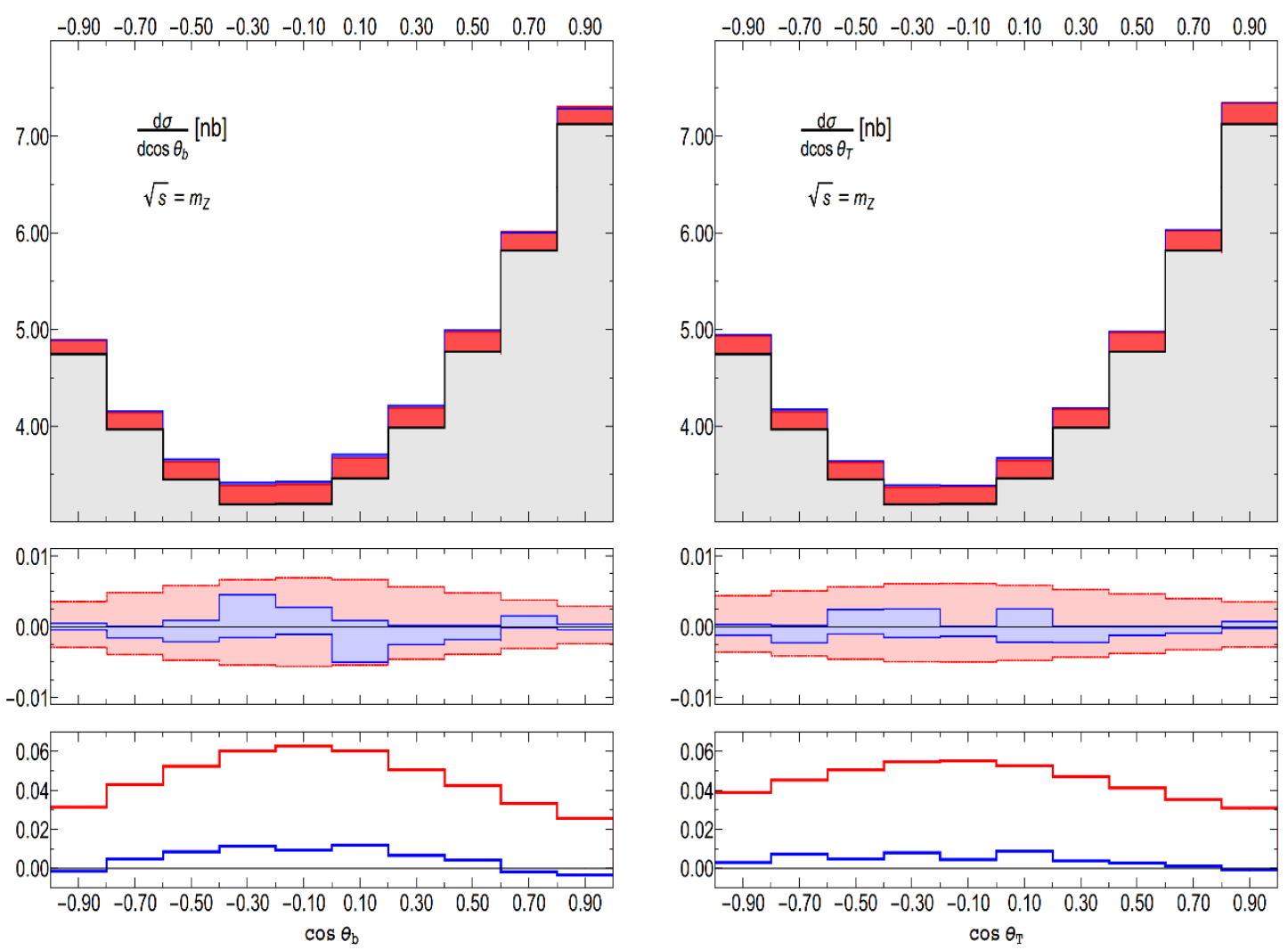

Figure 5. The distribution of $\cos \theta_{b}$ (plots on the left) and of $\cos \theta_{T}$ (plots on the right) at $\sqrt{s}=m_{Z}$. The upper panels show the respective distribution at LO (grey), NLO (red), and NNLO QCD (blue) for $\mu=m_{Z}$. The panels in the middle display the scale variations $\operatorname{NLO}\left(\mu^{\prime}\right) / \operatorname{NLO}\left(\mu=m_{Z}\right)-1$ (red band) and $\operatorname{NNLO}\left(\mu^{\prime}\right) / \operatorname{NNLO}\left(\mu=m_{Z}\right)-1$ (blue band) of the first and second order QCD corrections, where $m_{Z} / 2 \leq \mu^{\prime} \leq 2 m_{Z}$. The lower panels show the ratios $d \sigma_{1} / d \sigma_{\mathrm{LO}}$ (red) and $d \sigma_{2} / d \sigma_{\mathrm{LO}}$ (blue) for $\mu=m_{Z}$.

\subsection{Approaching the limit of massless $b$ quarks}

Next we compute the second-order correction to the $b$-quark forward-backward asymmetry for a sequence of decreasing values of $m_{b}$. This allows us to compare with the results of $[18,19]$ obtained for $m_{b}=0$. In order to conform to the calculation of [19] we neglect now, as was done in [19], the singlet and the triangle contributions. Thus we take into account only the non-singlet contributions to $(2.11)$ which we denote by $A_{2}^{\mathrm{NS}}$. We recall here the classification of the various second-order contributions done in section 3 that is in accord with [19].

It was shown in [19] that the second-order correction $A_{2}^{\mathrm{NS}}$ becomes singular for $m_{b} \rightarrow 0$ due to a logarithmic singularity that arises in the phase-space integration of the symmetric $E$-term in the triple-collinear regions. As mentioned above, the $E$-term is the color subleading contribution to the squared matrix element of the $b \bar{b} b \bar{b}$ final state. It consists of the interference terms listed in (3.2). It was also shown in ref. [19] that $A_{2}^{\mathrm{NS}}$ can be 
decomposed as follows:

$$
A_{2}^{\mathrm{NS}}=\widehat{A}_{2}-\int E_{S},
$$

where $\int E_{S}$ denotes the phase-space integral over the symmetric E-term that contains a term $\propto \alpha_{s}^{2} \ln \left(s / m_{b}\right)$ and $\widehat{A}_{2}$ is finite in the limit $m_{b} \rightarrow 0$. We recall that the $b \bar{b} b \bar{b}$ diagram contributions to $A_{2}^{\mathrm{NS}}$ are multiplied by a factor of 2 (cf. section 3 ).

The term $\widehat{A}_{2}$ was calculated for $m_{b}=0$ and for the quark axis definition in $[18,19]$ and for the thrust axis definition in [19]. Here we compute $\widehat{A}_{2}$, respectively the coefficient

$$
\widehat{a}_{2}=-\left(\frac{2 \pi}{\alpha_{s}}\right)^{2} \widehat{A}_{2}
$$

for a sequence of decreasing $b$-quark mass values between $m_{b}=4.89 \mathrm{GeV}$ and $m_{b}=1 \mathrm{GeV}$. We choose the renormalization scale to be $\mu=m_{Z}$ which was apparently also chosen in [19]. We compute $\widehat{a}_{2}$ both for the quark and thrust axis definition of $A_{\mathrm{FB}}$. The results are shown by the red solid triangle points in the left and right plots of figure 6 . In order to extrapolate $\widehat{a}_{2}$ to $m_{b}=0$ we perform a fit using the ansatz

$$
c_{0}+c_{1} z+c_{2} z \ln z^{2}
$$

where $z=\left(m_{b} / m_{Z}\right)$. This ansatz is motivated by the leading mass terms of the NNLO corrections in the limit $z \rightarrow 0$. The coefficient $c_{0}$ is the value of $\widehat{a}_{2}$ at $m_{b}=0$. We obtain

$$
\text { quark axis: } c_{0}=36.40 \pm 1.70, \quad \text { thrust axis: } c_{0}=24.83 \pm 1.78,
$$

which agree within errors with the values

$$
\widehat{a}_{2 b}\left(m_{b}=0\right)=38.5, \quad \widehat{a}_{2 T}\left(m_{b}=0\right)=26.74
$$

for the quark $[18,19]$ and thrust axis definition [19], respectively.

If one wants to compare the size of the QCD corrections to $A_{\mathrm{FB}}$ for a massive and a massless $b$-quark, one should compare the massless order $\alpha_{s}^{2}$ correction coefficients (4.8) of $[18,19]$ with the respective coefficients shown in figure 6 for non-zero $m_{b}$, rather than comparing with the coefficients $a_{2}$ given in table 2 , because the latter contain also singlet and triangle contributions. Figure 6 shows that both for the quark and for the thrust axis definition, the second-order corrections are smaller in magnitude for massive quarks than for massless ones. For $m_{b}=4.89 \mathrm{GeV}$ we obtain

$$
\widehat{a}_{2 b}\left(m_{b}=4.89 \mathrm{GeV}\right)=23.31, \quad \widehat{a}_{2 T}\left(m_{b}=4.89 \mathrm{GeV}\right)=18.43 .
$$

The magnitude of the second-order corrections decreases with increasing quark mass. This holds true also for the first-order corrections, as exemplified by comparing the values of $a_{1}$ for $m_{b}=4.89 \mathrm{GeV}$ listed in table 2 with the values $a_{1 b}=2$ and $a_{1 T}=1.787$ for $m_{b}=0$. This is in accord with the basic physical fact that a massive (anti)quark is more inert than a massless one in radiating off partons, and hence less affected by changes of its direction with respect to the leading-order quark antiquark configuration. 

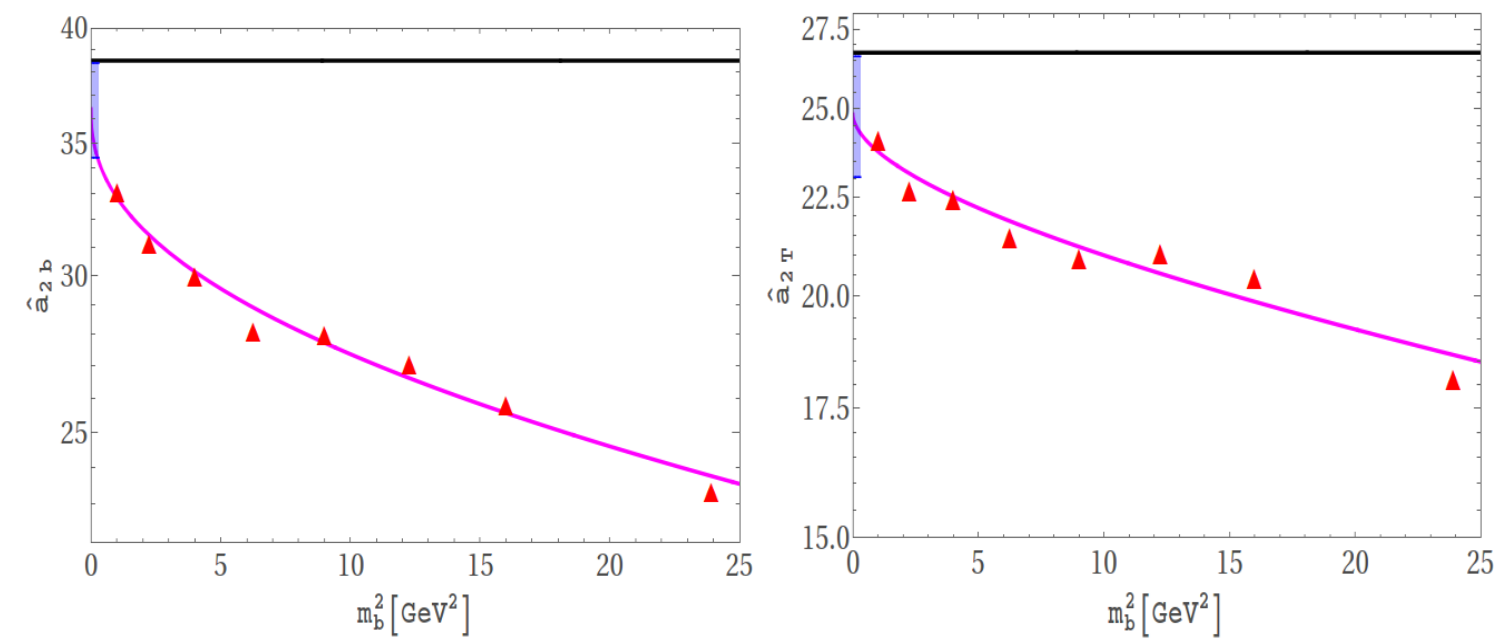

Figure 6. Left plot: the solid red triangles are the values of the order $\alpha_{s}^{2}$ correction coefficients $\widehat{a}_{2}$ defined in (4.5) for the quark axis definition for a sequence of $b$-quark mass values and $\mu=m_{Z}$. The solid red curve is obtained by a fit using the ansatz (4.6). The horizontal solid black line is the value for $m_{b}=0$ computed in $[18,19]$. The shaded blue vertical line is the $1 \sigma$ uncertainty of the value of $\widehat{a}_{2}$ at $m_{b}=0$ resulting from the fit. Right plot: same as left plot, but for the thrust axis definition of $A_{\mathrm{FB}}$. Here the solid black line is the value of $\widehat{a}_{2}$ at $m_{b}=0$ computed in [19].

Finally, as an aside, we close this section by pointing out the difference between the various contributions to the QCD corrections to the $b$-quark asymmetry and the top-quark forward-backward asymmetry in $t \bar{t}$ production computed in [14, 15]. Contrary to the b-quark asymmetry at the $\mathrm{Z}$ peak, the first and second-order QCD corrections to the topquark asymmetry are dominated, for energies $\sqrt{s} \lesssim 1 \mathrm{TeV}$ where $m_{t} / \sqrt{s}$ is not very small, by the two-parton contributions.

\subsection{Discussion}

The QCD corrections to the $b$-quark $A_{\mathrm{FB}}$ determined above (or those determined in the calculations $[18,19]$ for $m_{b}=0$ ) cannot be applied directly to the analysis of experiments. In the measurements of the $b$-quark asymmetry reviewed in $[1,2]$, the thrust axis was used to define the forward and backward hemispheres. In our computation the thrust axis is defined for partonic final states, but the hadronization of partons causes a smearing of this axis. In addition, a bias in the topology of the events is introduced by the experimental selection and analysis method towards two-jet final states which causes additional uncertainties [23, 24].

A proper discussion of these issues is beyond the scope of this paper. Here we only compare the QCD corrections computed above with those that were taken into account in $[1,2,24]$. These analyses aimed at determining a pseudo-observable: the bare $b$-quark $Z$-pole asymmetry $A_{\mathrm{FB}}^{0, b}$ from the measured asymmetry $A_{\mathrm{FB}, \exp }^{b, T}$ with a procedure described in $[23,24]$. First, $A_{\mathrm{FB}, \exp }^{b, T}$ was corrected for QCD effects as follows (cf. (4.3)):

$$
A_{\mathrm{FB}, \exp }^{b, T}=\left[1-a_{1 T} \frac{\alpha_{s}}{2 \pi}-a_{2 T}\left(\frac{\alpha_{s}}{2 \pi}\right)^{2}\right]\left(A_{\mathrm{FB}}^{0, b}\right)_{\exp } \equiv\left(1-C_{\mathrm{QCD}}^{T}\right)\left(A_{\mathrm{FB}}^{0, b}\right)_{\exp }
$$


The QCD corrected "experimental" asymmetry $\left(A_{\mathrm{FB}}^{0, b}\right)_{\exp }$ was then further corrected for higher order electroweak corrections like photon exchange, $Z \gamma$ interference, and other photonic corrections (cf. for instance, $[9,10]$ ) before a value of the bare asymmetry $A_{\mathrm{FB}}^{0, b}$ was deduced. In this way ref. [2] obtained the experimental value for the pseudo-observable

$$
A_{F B}^{0, b}=0.0992 \pm 0.0016,
$$

where the error refers to experimental and theoretical uncertainties. The pull between (4.11) and the value $A_{F B}^{0, b}=0.1038$ obtained by a combined fit to all SM precision observables [2] is $2.9 \sigma$.

The QCD correction factor defined in (4.10) and used in [2] was obtained as follows [23, 24]. For the order $\alpha_{s}$ correction the value $a_{1 T}=1.54$ was $\operatorname{taken}^{3}$ that was computed in [13] for $m_{b}=4.5 \mathrm{GeV}$. For the second-order QCD correction coefficient the value $a_{2}^{T}\left(m_{b}=0\right)=23.72$ was used. This number is obtained by adding to the massless result of [19] (cf. (4.8)) the two-loop $b \bar{b}$ triangle contribution. (The sign of this contribution to $a_{2}^{T}$ is opposite to that of (4.8).) The QCD correction factor determined in [24] is $\left(1-C_{\mathrm{QCD}}^{T}\right)=0.9646 \pm 0.0063$ where the error includes estimates of hadronization effects. Our thrust axis correction factor $\left(1+A_{1}+A_{2}\right)=0.9608 \pm 0.0025$ given in table 1 , where the error is due to scale uncertainties only, agrees with that factor within the uncertainties. Our central value is smaller than 0.9646 by $0.4 \%$. Our correction changes the value of the pseudo-observable $A_{F B}^{0, b}$ to $0.0996 \pm 0.0016$. Thus the pull between $A_{F B}^{0, b}$ and the SM fit cited above is slightly decreased, namely from $2.9 \sigma$ to $2.6 \sigma$.

We recall that our value of the QCD correction factor was obtained by taking into account all second-order QCD contributions discussed in section 3. If one neglects the singlet contributions, that is, if one uses the value of $\widehat{a}_{2 T}\left(m_{b}=4.89 \mathrm{GeV}\right)$ given in (4.9) and adds the $b \bar{b}$ and $b \bar{b} g$ triangle contributions, we get for the thrust axis correction factor $\left(1+A_{1}+A_{2}\right)=0.9659 \pm 0.0023$. This value is not significantly larger than the correction factor used in [24] and cited above.

\section{Conclusions}

We have computed the second-order QCD corrections to the $b$-quark forward-backward asymmetry in $e^{+} e^{-} \rightarrow b \bar{b}$ collisions at the $Z$ boson resonance. The mass of the $b$ quark was fully taken into account. We have determined these corrections both for using the quark and the thrust axis in defining the forward and backward hemisphere. We have computed also the distributions of these axes with respect to the electron beam. The complete order $\alpha_{s}^{2}$ corrections to the $b$-quark asymmetry, that is, the sum of the flavor non-singlet, flavor singlet, and triangle contributions are significant; they amount to $43 \%$ and $37 \%$ of the order $\alpha_{s}$ corrections for the quark and thrust axis definition, respectively. If one neglects the singlet contributions, as was done in previous calculations for massless $b$ quarks $[18,19]$, then the second-order QCD corrections for $m_{b} \neq 0$ are smaller in magnitude than the

\footnotetext{
${ }^{3}$ This value is almost the same as the value $a_{1 T}=1.53$ given in table 2 . We recall that we use a slightly larger $b$-quark mass. Moreover, we determine the thrust axis by classifying the final states according to the moduli of their three-momenta rather than their energies as in [13].
} 
corresponding corrections for $m_{b}=0$. This is expected on general physical grounds. We have also demonstrated that by decreasing the value of the $b$-quark mass we can approach with our computational set-up the massless order $\alpha_{s}^{2}$ results of $[18,19]$.

As emphasized above our results cannot be applied directly to the analysis of existing measurements of the $b$-quark asymmetry. We have compared the magnitude of the secondorder QCD corrections for $m_{b} \neq 0$ with those used in previous analyses that deduced a bare $b$-quark asymmetry $A_{\mathrm{FB}}^{0, b}$ from the measured one. If one takes into account the complete massive order $\alpha_{s}^{2}$ correction then the value of the bare asymmetry (4.11) increases slightly, which reduces the pull between this pseudo-observable and the value of the standard model fit from $2.9 \sigma$ to $2.6 \sigma$.

As a future application of our computational set-up one may consider the determination of the order $\alpha_{s}^{2}$ corrections to the forward-backward asymmetry for two $b$-jet final states, for which one expects a decrease of the magnitude of the QCD corrections.

\section{Acknowledgments}

L. Chen acknowledges support by a scholarship from the China Scholarship Council (CSC).

D. Heisler was supported by Deutsche Forschungsgemeinschaft through Graduiertenkolleg GRK 1675.

Open Access. This article is distributed under the terms of the Creative Commons Attribution License (CC-BY 4.0), which permits any use, distribution and reproduction in any medium, provided the original author(s) and source are credited.

\section{References}

[1] SLD Electroweak Group, DelPhi, ALEPH, SLD, SLD Heavy Flavour Group, OPAL, LEP Electroweak Working Group and L3 collaborations, S. Schael et al., Precision electroweak measurements on the $Z$ resonance, Phys. Rept. 427 (2006) 257 [hep-ex/0509008] [INSPIRE].

[2] Tevatron Electroweak Working Group, CDF, Delphi, Sld Electroweak and Heavy Flavour Groups, AlePh, LeP Electroweak Working Group, SLD, OPAL, D0 and L3 collaborations, L.E.W. Group, Precision Electroweak Measurements and Constraints on the Standard Model, arXiv:1012.2367 [INSPIRE].

[3] ECFA/DESY LC Physics Working Group collaboration, J.A. Aguilar-Saavedra et al., TESLA: The Superconducting electron positron linear collider with an integrated $x$-ray laser laboratory. Technical design report. Part 3. Physics at an $e^{+} e^{-}$linear collider, hep-ph/0106315 [INSPIRE].

[4] H. Baer et al., The International Linear Collider Technical Design Report - Volume 2: Physics, arXiv:1306.6352 [INSPIRE].

[5] TleP Design Study Working Group collaboration, M. Bicer et al., First Look at the Physics Case of TLEP, JHEP 01 (2014) 164 [arXiv:1308.6176] [InSPIRE].

[6] R. Hawkings and K. Mönig, Electroweak and CP-violation physics at a linear collider Z factory, Eur. Phys. J. direct C 1 (1999) 8 [hep-ex/9910022] [InSPIRE]. 
[7] J. Erler, S. Heinemeyer, W. Hollik, G. Weiglein and P.M. Zerwas, Physics impact of GigaZ, Phys. Lett. B 486 (2000) 125 [hep-ph/0005024] [INSPIRE].

[8] M. Böhm et al., Forward-Backward Asymmetries, in: CERN Yellow Report $Z$ Physics at LEP 1, G. Altarelli et al. eds., CERN 89-08 (1989).

[9] D. Yu. Bardin et al., ZFITTER v.6.21: A Semianalytical program for fermion pair production in $e^{+} e^{-}$annihilation, Comput. Phys. Commun. 133 (2001) 229 [hep-ph/9908433] [INSPIRE].

[10] A. Freitas and K. Mönig, Corrections to quark asymmetries at LEP, Eur. Phys. J. C 40 (2005) 493 [hep-ph/0411304] [INSPIRE].

[11] J. Jersak, E. Laermann and P.M. Zerwas, Electroweak Production of Heavy Quarks in $e^{+} e^{-}$ Annihilation, Phys. Rev. D 25 (1982) 1218 [Erratum ibid. D 36 (1987) 310] [INSPIRE].

[12] A.B. Arbuzov, D. Yu. Bardin and A. Leike, Analytic final state corrections with cut for $e^{+} e^{-} \rightarrow$ massive fermions, Mod. Phys. Lett. A 7 (1992) 2029 [Erratum ibid. A 9 (1994) 1515] [INSPIRE].

[13] A. Djouadi, B. Lampe and P.M. Zerwas, A Note on the QCD corrections to forward-backward asymmetries of heavy quark jets in Z decays, Z. Phys. C 67 (1995) 123 [hep-ph/9411386] [INSPIRE].

[14] J. Gao and H.X. Zhu, Top Quark Forward-Backward Asymmetry in $e^{+} e^{-}$Annihilation at Next-to-Next-to-Leading Order in QCD, Phys. Rev. Lett. 113 (2014) 262001 [arXiv:1410.3165] [INSPIRE].

[15] L. Chen, O. Dekkers, D. Heisler, W. Bernreuther and Z.-G. Si, Top-quark pair production at next-to-next-to-leading order QCD in electron positron collisions, JHEP 12 (2016) 098 [arXiv: 1610.07897] [INSPIRE].

[16] M. Czakon, P. Fiedler and A. Mitov, Resolving the Tevatron Top Quark Forward-Backward Asymmetry Puzzle: Fully Differential Next-to-Next-to-Leading-Order Calculation, Phys. Rev. Lett. 115 (2015) 052001 [arXiv: 1411.3007] [INSPIRE].

[17] G. Altarelli and B. Lampe, Second order QCD corrections to heavy quark forward-backward asymmetries, Nucl. Phys. B 391 (1993) 3 [INSPIRE].

[18] V. Ravindran and W.L. van Neerven, Second order QCD corrections to the forward-backward asymmetry in $e^{+} e^{-}$collisions, Phys. Lett. B 445 (1998) 214 [hep-ph/9809411] [INSPIRE].

[19] S. Catani and M.H. Seymour, Corrections of $O\left(\alpha_{s}^{2}\right)$ to the forward backward asymmetry, JHEP 07 (1999) 023 [hep-ph/9905424] [INSPIRE].

[20] S. Weinzierl, The Forward-backward asymmetry at NNLO revisited, Phys. Lett. B 644 (2007) 331 [hep-ph/0609021] [inSPIRE].

[21] A. Banfi, G.P. Salam and G. Zanderighi, Infrared safe definition of jet flavor, Eur. Phys. J. C 47 (2006) 113 [hep-ph/0601139] [INSPIRE].

[22] W. Bernreuther et al., Two-Parton Contribution to the Heavy-Quark Forward-Backward Asymmetry in NNLO QCD, Nucl. Phys. B 750 (2006) 83 [hep-ph/0604031] [inSPIRE].

[23] LEP Heavy Flavor Working Group collaboration, D. Abbaneo et al., QCD corrections to the forward-backward asymmetries of $c$ and $b$ quarks at the $Z$ pole, Eur. Phys. J. C 4 (1998) 185 [INSPIRE]. 
[24] LEP/SLD heAvy FLAVOuR WORKIng Group collaboration, Final Input Parameters for the LEP/SLD Heavy Flavour Analyses, LEPHF/2001-01 (2001), http://lepewwg.web.cern.ch/LEPEWWG/heavy/.

[25] W. Bernreuther et al., Two-loop QCD corrections to the heavy quark form-factors: The Vector contributions, Nucl. Phys. B 706 (2005) 245 [hep-ph/0406046] [InSPIRE].

[26] W. Bernreuther et al., Two-loop QCD corrections to the heavy quark form-factors: Axial vector contributions, Nucl. Phys. B 712 (2005) 229 [hep-ph/0412259] [INSPIRE].

[27] W. Bernreuther, R. Bonciani, T. Gehrmann, R. Heinesch, T. Leineweber and E. Remiddi, Two-loop QCD corrections to the heavy quark form-factors: Anomaly contributions, Nucl. Phys. B 723 (2005) 91 [hep-ph/0504190] [INSPIRE].

[28] E. Farhi, A QCD Test for Jets, Phys. Rev. Lett. 39 (1977) 1587 [inSPIRE].

[29] S. Brandt, C. Peyrou, R. Sosnowski and A. Wroblewski, The Principal axis of jets. An Attempt to analyze high-energy collisions as two-body processes, Phys. Lett. 12 (1964) 57 [INSPIRE].

[30] S. Brandt and H.D. Dahmen, Axes and Scalar Measures of Two-Jet and Three-Jet Events, Z. Phys. C 1 (1979) 61 [INSPIRE].

[31] T. Sjöstrand, S. Mrenna and P.Z. Skands, PYTHIA 6.4 Physics and Manual, JHEP 05 (2006) 026 [hep-ph/0603175] [INSPIRE].

[32] R.K. Ellis, D.A. Ross and A.E. Terrano, The Perturbative Calculation of Jet Structure in $e^{+} e^{-}$Annihilation, Nucl. Phys. B 178 (1981) 421 [INSPIRE].

[33] Particle Data Group collaboration, C. Patrignani et al., Review of Particle Physics, Chin. Phys. C 40 (2016) 100001 [InSPIRE]. 